\title{
Fractional operators for the Wright hypergeometric matrix functions
}

\section{Abdalla $a^{1,2^{*}}$}

\section{"Correspondence:}

moabdalla@kku.edu.sa

${ }^{1}$ Mathematics Department, Faculty

of Science, King Khalid University,

Abha, Saudi Arabia

${ }^{2}$ Department of Mathematics,

Faculty of Science, South Valley

University, Qena, Egypt

\begin{abstract}
In this paper, we contribute to the results of Bakhet et al. (Integral Transforms Spec. Funct. 30:138-156, 2019) by applying fractional operators to the Wright hypergeometric matrix functions. We give matrix recurrence relations and integral formulas for the Wright hypergeometric matrix functions. We also clarify particular cases of the main results.
\end{abstract}

MSC: $15 \mathrm{~A} 15 ; 33 \mathrm{C} 45 ; 33 \mathrm{C} 05 ; 34 \mathrm{~A} 05$

Keywords: Wright hypergeometric matrix function; Fractional integral and differential operators; Matrix recurrence relation; Integral formulae

\section{Introduction}

The studies of one-variable hypergeometric functions are more than 200 years old. These functions appear in the works of Euler, Gauss, Riemann, and Kummer (see [2]). Their integral representations were studied by Barnes and Mellin, and their special properties by Schwarz and Goursat. The famous Gauss hypergeometric equation is ubiquitous in mathematical physics, engineering, and mathematical sciences as many well-known partial differential equations may be reduced to the Gauss equation via separation of variables (see, e.g., [3-6]). Especially, in the last two decades, several generalizations of the well-known special functions have been studied by many mathematicians. This fact has inspired many mathematicians for investigations of several generalizations of hypergeometric functions.

Recently, many authors (see, e.g., [1, 7-14]) proposed extensions of the classical generalized hypergeometric functions to the matrix framework. Particularly, the Wright hypergeometric matrix functions of one variable and incomplete Wright Gauss hypergeometric matrix functions by using the Pochhammer matrix symbol are investigated in [1]. Motivated mainly by this work, here we introduce the matrix functions $\mathcal{R}(w ; A, B ; v ; \lambda), w, v, \lambda \in$ $\mathbb{C}$, and $\mathcal{R}(w ; A, B ;-\mu ; \lambda), w, \mu, \lambda \in \mathbb{C}$, for parameter matrices $A$ and $B$ by applying fractional operators to the Wright hypergeometric matrix function and some their properties.

The outline of the paper is as follows. Section 2 gives some elementary definitions and notions of this work. In Sect. 3, we obtain the matrix functions $\mathcal{R}(w ; A, B ; v ; \lambda), w, v, \lambda \in \mathbb{C}$, and $\mathcal{R}(w ; A, B ;-\mu ; \lambda), w, \mu, \lambda \in \mathbb{C}$, for parameter matrices $A$ and $B$ by using fractional operators. We also discuss some properties of these functions. Matrix recurrence relations of

(c) The Author(s) 2020. This article is licensed under a Creative Commons Attribution 4.0 International License, which permits use sharing, adaptation, distribution and reproduction in any medium or format, as long as you give appropriate credit to the original author(s) and the source, provide a link to the Creative Commons licence, and indicate if changes were made. The images or other third party material in this article are included in the article's Creative Commons licence, unless indicated otherwise in a credit line to the material. If material is not included in the article's Creative Commons licence and your intended use is not permitted by statutory regulation or exceeds the permitted use, you will need to obtain permission directly from the copyright holder. To view a copy of this licence, visit http://creativecommons.org/licenses/by/4.0/. 
the Wright hypergeometric matrix function ${ }_{2} \mathbf{R}_{1}^{(\tau)}(A, B ; C ; z), z \in \mathbb{C}$, for parameter matrices $A, B$, and $C$ and some particular cases are given in Sect. 4. In Sect. 5, we establish some (presumably) new integral representations of the Wright hypergeometric matrix function ${ }_{2} \mathbf{R}_{1}^{(\tau)}(A, B ; C ; z)$. Finally, some concluding remarks are presented in Sect. 6.

\section{Notations and definitions}

Let $\mathbb{C}^{N \times N}$ denote the vector space containing all square matrices with $N$ rows and $N$ columns with complex number entries, and let $\mathcal{R}(z)$ and $\mathcal{I}(z)$ denote the real and imaginary parts of a complex number $z$, respectively. For any matrix $\mathrm{A}$ in $\mathbb{C}^{N \times N}, \sigma(A)$ is the spectrum of $A$, the set of all eigenvalues of $A$, and

$$
\alpha(A)=\max \{\mathcal{R}(z): z \in \sigma(A)\}, \quad \beta(A)=\min \{\mathcal{R}(z): z \in \sigma(A)\},
$$

where $\alpha(A)$ is referred to as the spectral abscissa of $A$, and $\alpha(-A)=-\beta(A)$. The square matrix $A$ is said to be positive stable if $\beta(A)>0$. By $I$ and 0 we denote the identity matrix and the null matrix in $\mathbb{C}^{N \times N}$, respectively. If $f(z)$ and $g(z)$ are holomorphic functions of the complex variable $z$ defined in an open subset $\Omega$ of the complex plane and $A$ is a matrix in $\mathbb{C}^{N \times N}$ such that $\sigma(A) \subset \Omega$, then from the properties of the matrix functional calculus (see $[9,15-17])$ it follows that

$$
f(A) g(A)=g(A) f(A)
$$

Furthermore, if $B$ in $\mathbb{C}^{N \times N}$ is a matrix for which $\sigma(B) \subset \Omega$ and if $A B=B A$, then

$$
f(A) g(B)=g(B) f(A) .
$$

The reciprocal gamma function denoted by $\Gamma^{-1}(z)=\frac{1}{\Gamma(z)}$ is an entire function of the complex variable $z$. Then the image of $\Gamma^{-1}(z)$ acting on $P$, denoted by $\Gamma^{-1}(A)$, is a welldefined matrix (see [18]).

The gamma matrix function $\Gamma(A)$ and the beta matrix function $\mathfrak{B}(A, B)$ have been defined in [9] as follows:

$$
\Gamma(A)=\int_{0}^{\infty} e^{-t} t^{A-I} d t, \quad t^{A-I}=\exp ((A-I) \ln t)
$$

and

$$
\mathfrak{B}(A, B)=\int_{0}^{1} t^{A-I}(1-t)^{B-I} d t
$$

By application of the matrix functional calculus, for $A$ in $\mathbb{C}^{N \times N}$ (see [9]) the Pochhammer symbol or shifted factorial is defined by

$$
(A)_{n}= \begin{cases}A(A+I) \cdots(A+(n-1) I)=\Gamma^{-1}(A) \Gamma(A+n I), & n \geq 1, \\ I, & n=0,\end{cases}
$$


under the condition that

$$
A+n I \quad \text { is invertible for all integers } n \geq 0 \text {. }
$$

Let $A$ and $B$ be commuting matrices in $\mathbb{C}^{N \times N}$ such that the matrices $A+n I, B+n I$, and $A+B+n I$ are invertible for every integer $n \geq 0$. Then we have [9]

$$
\mathfrak{B}(A, B)=\Gamma(A) \Gamma(B)[\Gamma(A+B)]^{-1} .
$$

Definition 2.1 (see [12]) Let $p$ and $q$ be finite positive integers. Then the generalized hypergeometric matrix function is given by the matrix power series

$$
F\left(A_{i}, B_{j} ; z\right)=\sum_{n \geq 0} \prod_{i=1}^{p}\left(A_{i}\right)_{n} \prod_{j=1}^{q}\left[\left(B_{j}\right)_{n}\right]^{-1} \frac{z^{n}}{n !} \quad(p=q+1,|z|<1)
$$

for commutative matrices $A_{i}, 1 \leq i \leq p$, and $B_{j}, 1 \leq j \leq q$, in $\mathbb{C}^{N \times N}$ such that

$B_{j}+n I$ are invertible for all integers $n \geq 0$.

If $p=2$ and $q=1$, then (7) reduces to the Gauss hypergeometric matrix function (see [7])

$$
{ }_{2} F_{1}\left(A_{1}, A_{2} ; B_{1} ; z\right)=\sum_{n=0}^{\infty}\left(A_{1}\right)_{n}\left(A_{2}\right)_{n}\left[\left(B_{1}\right)_{n}\right]^{-1} \frac{z^{n}}{n !} .
$$

Definition 2.2 ([1]) Let $A, B$, and $C$ be positive stable matrices in $\mathbb{C}^{N \times N}$ satisfying condition (5). Then the Wright hypergeometric matrix function is defined by

$$
{ }_{2} \mathbf{R}_{1}^{(\tau)}(A, B ; C ; z):=\Gamma^{-1}(B) \Gamma(C) \sum_{n=0}^{\infty}(A){ }_{n} \Gamma^{-1}(C+\tau n I) \Gamma(B+\tau n I) \frac{z^{n}}{n !},
$$

where $\tau \in \mathbb{R}_{+}=(0, \infty)$.

Remark If $\tau=1$, then (9) reduces to the well-known hypergeometric matrix function ${ }_{2} F_{1}$ defined in (8).

Theorem 2.1 ([1]) Let $A, B$, and $C$ be positive stable matrices in $\mathbb{C}^{N \times N}$ such that

$$
\beta(C)-\alpha(A)-\alpha(B)>0 .
$$

Then the series ${ }_{2} \mathbf{R}_{1}^{(\tau)}(A, B ; C ; z)$ defined in (9) converges absolutely for $|z|=1$, where $\tau \in$ $\mathbb{R}_{+}=(0, \infty)$.

The Riemann-Liouville fractional integral of order $v$ such that $\operatorname{Re}(v)>0$ is defined as $[16,19,20]$

$$
\left(\mathbf{I}^{v} f\right)(w)=\frac{1}{\Gamma(v)} \int_{0}^{w}(w-u)^{v-1} f(u) d u
$$


and the fractional differential operator of order $\mu$ such that $\operatorname{Re}(\mu)>0$ is defined as

$$
\mathbf{D}^{\mu} f(w)=\mathbf{I}^{n-\mu} \mathbf{D}^{n} f(w), \quad \mathbf{D}=\frac{d}{d w}
$$

where $n$ is the smallest integer such that $n>\operatorname{Re}(\mu)$.

Lemma $2.1([1,15,16])$ Let $A$ be a positive stable matrix in $\mathbb{C}^{N \times N}$ Then the RiemannLiouville fractional integral of order $v$ such that $\operatorname{Re}(v)>0$ can be written as

$$
\mathbf{I}^{v}\left(w^{A-I}\right)=\Gamma(A) \Gamma^{-1}(A+v I) w^{A+(v-1) I} .
$$

The Laplace transform of the function $f(\zeta)$ is defined as [21]

$$
\mathcal{L}\{f(\zeta)\}=\int_{0}^{\infty} e^{-s \zeta} f(\zeta) d \zeta, \quad \operatorname{Re}(s)>0
$$

\section{Fractional operators and the Wright-type hypergeometric matrix function}

Consider the matrix function

$$
\begin{aligned}
f(w) & =\Gamma^{-1}(B) \sum_{k=0}^{\infty} \Gamma(B+k I) \frac{(A)_{k}(\lambda w)^{k}}{(k !)^{2}} \\
& ={ }_{2} F_{1}(A, B ; I ; \lambda w), \quad w, \lambda \in \mathbb{C},|\lambda w|<1,
\end{aligned}
$$

where $A$ and $B$ are positive stable matrices in $\mathbb{C}^{N \times N}$ such that

$$
B+n I \text { are invertible for all integers } n \geq 0 \text {. }
$$

Applying the fractional integral operator (11) of order $v$ to $f(w)$, we get

$$
\begin{aligned}
\mathbf{I}^{v} f(w) & =\frac{1}{\Gamma(v)} \int_{0}^{w}\left((w-u)^{v-1} f(u)\right) d u \\
& =\frac{1}{\Gamma(v)} \int_{0}^{w}\left((w-u)^{v-1} \Gamma^{-1}(B) \sum_{k=0}^{\infty} \Gamma(B+k I) \frac{(A)_{k}(\lambda u)^{k}}{(k !)^{2}}\right) d u \\
& =\frac{w^{v}}{\Gamma(v+1)}\left\{\Gamma(v+1) \Gamma^{-1}(B) \sum_{k=0}^{\infty} \Gamma(B+k I) \frac{(A)_{k}(\lambda w)^{k}}{\Gamma(v+1+k) k !}\right\},
\end{aligned}
$$

which we can easily write in the following form:

$$
\begin{gathered}
\frac{w^{v}}{\Gamma(v+1)}{ }_{2} \mathbf{R}_{1}^{(1)}(A, B ;(v+1) I ; \lambda w) \quad(\tau=1) \\
=\frac{w^{v}}{\Gamma(v+1)}{ }_{2} F_{1}(A, B ;(v+1) I ; \lambda w) .
\end{gathered}
$$

Here we denote $(16)$ as $\mathcal{R}(w ; A, B ; v ; \lambda)$, that is,

$$
\mathcal{R}(w ; A, B ; v ; \lambda)=\frac{w^{v}}{\Gamma(v+1)}{ }_{2} \mathbf{R}_{1}^{(1)}(A, B ;(v+1) I ; \lambda w)
$$




$$
=\frac{w^{v}}{\Gamma(v+1)}{ }_{2} F_{1}(A, B ;(v+1) I ; \lambda w) .
$$

Now, applying the fractional differential operator (12) of order $\mu$ to $f(w)$ defined in (15), we get

$$
\begin{aligned}
\mathbf{D}^{\mu} f(w) & =\left(\frac{d}{d w}\right)^{n}\left(\mathbf{I}^{n-\mu} \Gamma^{-1}(B) \sum_{k=0}^{\infty} \Gamma(B+k I) \frac{(A)_{k}(\lambda w)^{k}}{(k !)^{2}}\right) \\
& =\mathbf{D}^{n}\left[w^{n-\mu} \Gamma^{-1}(B)\left\{\sum_{k=0}^{\infty} \Gamma(B+k I) \frac{(A)_{k}(\lambda w)^{k}}{\Gamma(1+n-\mu+k) k !}\right\}\right],
\end{aligned}
$$

which yields

$$
\begin{aligned}
\mathbf{D}^{\mu} f(w) & =\frac{w^{-\mu}}{\Gamma(1-\mu)}{ }_{2} \mathbf{R}_{1}^{(1)}(A, B ;(1-\mu) I ; \lambda w) \quad(\tau=1) \\
& =\frac{w^{-\mu}}{\Gamma(1-\mu)}{ }_{2} F_{1}(A, B ;(1-\mu) I ; \lambda w) .
\end{aligned}
$$

We denote (18) as

$$
\begin{aligned}
\mathcal{R}(w ; A, B ;-\mu ; \lambda) & =\frac{w^{-\mu}}{\Gamma(1-\mu)}{ }_{2} \mathbf{R}_{1}^{(1)}(A, B ;(1-\mu) I ; \lambda w) \\
& =\frac{w^{-\mu}}{\Gamma(1-\mu)}{ }_{2} F_{1}(A, B ;(1-\mu) I ; \lambda w) .
\end{aligned}
$$

\subsection{Some properties of $\mathcal{R}(w ; A, B ; \nu ; \lambda)$ and $\mathcal{R}(w ; A, B ;-\mu ; \lambda)$}

In this subsection, we study some of the main properties of the matrix functions $\mathcal{R}(w ; A, B ; \nu ; \lambda)$ and $\mathcal{R}(w ; A, B ;-\mu ; \lambda)$ by the following theorems.

Theorem 3.1 Let $A$ and $B$ be positive stable matrices in $\mathbb{C}^{N \times N}$ with $B+n I$ invertible for all integers $n \geq 0$, and let $\lambda, v, \mu \in \mathbb{C}$ be such that $|\lambda w|<1$ and $\operatorname{Re}(\mu)<1$. Then

$$
\begin{aligned}
& \mathbf{I}^{\gamma} \mathcal{R}(w ; A, B ; v ; \lambda)=\mathcal{R}(w ; A, B ; v+\gamma ; \lambda), \\
& \mathbf{D}^{\gamma} \mathcal{R}(w ; A, B ; v ; \lambda)=\mathcal{R}(w ; A, B ; v-\gamma ; \lambda), \\
& \mathbf{I}^{\gamma} \mathcal{R}(w ; A, B ;-\mu ; \lambda)=\mathcal{R}(w ; A, B ; \gamma-\mu ; \lambda), \\
& \mathbf{D}^{\gamma} \mathcal{R}_{w}(A, B ; v ; \lambda)=\mathcal{R}(w ; A, B ;-(\gamma+\mu) ; \lambda) .
\end{aligned}
$$

Proof From (11) and the left-hand side of (20) we get

$$
\begin{aligned}
\mathbf{I}^{\gamma} \mathcal{R}(w ; A, B ; v ; \lambda) & =\frac{1}{\Gamma(\gamma)} \int_{0}^{w}(w-u)^{\gamma-1} \mathcal{R}(u ; A, B ; v ; \lambda) d u \\
& =\frac{1}{\Gamma(\gamma)} \int_{0}^{w}(w-u)^{\gamma-1}\left(\frac{u^{v}}{\Gamma(v+1)}{ }_{2} R_{1}^{(1)}(A, B ; v+1 ; \lambda u)\right) d u \\
& =\frac{1}{\Gamma(\gamma)} \int_{0}^{w}(w-u)^{\gamma-1}\left(u^{v} \Gamma^{-1}(B) \sum_{k=0}^{\infty} \frac{(A)_{k} \Gamma(B+I k)(\lambda u)^{k}}{\Gamma(v+1+k) k !}\right) d u .
\end{aligned}
$$


Putting $u=z w$, it follows that

$$
\begin{aligned}
\mathbf{I}^{\gamma} & \mathcal{R}_{w}(A, B ; v ; \lambda) \\
\quad= & \frac{1}{\Gamma(\gamma)} \int_{0}^{1}(1-z)^{\gamma-1} w^{\gamma-1}\left((z w)^{v} \Gamma^{-1}(B) \sum_{k=0}^{\infty} \frac{(A)_{k} \Gamma(B+I k)(\lambda z w)^{k}}{\Gamma(v+1+k) k !}\right) w d z \\
\quad= & \frac{w^{\gamma+v}}{\Gamma(\gamma+v+1)}{ }_{2} R_{1}^{(1)}(A, B ; \gamma+v+1 ; \lambda w)=\mathcal{R}(w ; A, B ; \gamma+v ; \lambda) .
\end{aligned}
$$

This is the proof of (20).

From (12) and the left-hand side of (21) we obtain

$$
\begin{aligned}
\mathbf{D}^{\gamma} \mathcal{R}(w ; A, B ; v ; \lambda) & =\mathbf{D}^{n}\left(\mathbf{I}^{n-\gamma} \mathcal{R}(w ; A, B ; \nu ; \lambda)\right) \\
& =\mathbf{D}^{n}(\mathcal{R}(w ; A, B ; n-\gamma+v ; \lambda)) \\
& =\mathbf{D}^{n}\left(\frac{w^{n-\gamma+v}}{\Gamma(n-\gamma+v+1)}{ }_{2} R_{1}^{(1)}(A, B ; n-\gamma+v+1 ; \lambda w)\right) \\
& =\frac{w^{v-\gamma}}{\Gamma(\nu-\gamma+1)}{ }_{2} R_{1}^{(1)}(A, B ; \nu-\gamma+1 ; \lambda w) \\
& =\mathcal{R}(w ; A, B ; \nu-\gamma ; \lambda),
\end{aligned}
$$

which is (21).

Also, from (11) and the left-hand side of (22) we get

$$
\begin{aligned}
\mathbf{I}^{\gamma} \mathcal{R}(w ; A, B ;-\mu ; \lambda) & =\frac{1}{\Gamma(\gamma)} \int_{0}^{w}(w-u)^{\gamma-1} \mathcal{R}(u ; A, B ;-\mu ; \lambda) d u \\
& =\frac{1}{\Gamma(\gamma)} \int_{0}^{w}(w-u)^{\gamma-1}\left(u^{-\mu} \Gamma^{-1}(B) \sum_{k=0}^{\infty} \frac{(A)_{k} \Gamma(B+I k)(\lambda u)^{k}}{\Gamma(1-\mu+k) k !}\right) d u
\end{aligned}
$$

which, upon substituting $u=z w$, yields

$$
\begin{aligned}
& \mathbf{I}^{\gamma} \mathcal{R}(w ; A, B ;-\mu ; \lambda) \\
& \quad=\frac{1}{\Gamma(\gamma)} \int_{0}^{1}(1-z)^{\gamma-1} w^{\gamma-1}\left((z w)^{-\mu} \Gamma^{-1}(B) \sum_{k=0}^{\infty} \frac{(A)_{k} \Gamma(B+I k)(\lambda z w)^{k}}{\Gamma(1-\mu+k) k !}\right) w d x \\
& \quad=\frac{w^{\gamma-\mu}}{\Gamma(\gamma-\mu+1)}{ }_{2} R_{1}^{(1)}(A, B ; \gamma-\mu+1 ; \lambda w)=\mathcal{R}(w ; A, B ; \gamma-\mu ; \lambda) .
\end{aligned}
$$

This is the proof of (22). From (12) and the left-hand side of (23) we get

$$
\begin{aligned}
\mathbf{D}^{\gamma} \mathcal{R}(w ; A, B ;-\mu ; \lambda) & =\mathbf{D}^{n}\left(\mathbf{I}^{n-\gamma} \mathcal{R}(w ; A, B ;-\mu ; \lambda)\right) \\
& =\mathbf{D}^{n}(\mathcal{R}(w ; A, B ; n-\gamma-\mu ; \lambda)) \\
& =\mathbf{D}^{n}\left(\frac{w^{n-\gamma-\mu}}{\Gamma(n-\gamma-\mu+1)}{ }_{2} R_{1}^{(1)}(A, B ; n-\gamma-\mu+1 ; \lambda w)\right) \\
& =\frac{w^{-\mu-\gamma}}{\Gamma(-\mu-\gamma+1)}{ }_{2} R_{1}^{(1)}(A, B ;-\mu-\gamma+1 ; \lambda w)
\end{aligned}
$$




$$
=\mathcal{R}(w ; A, B ;-\mu-\gamma ; \lambda),
$$

which leads to (24).

Theorem 3.2 Let $C$ be a positive stable matrix in $\mathbb{C}^{N \times N}$. Then the Laplace transforms of $\mathcal{R}(w ;-n I, C+(n-1) I ; v ; \lambda)$ and $\mathcal{R}(w ;-n I, C+(n-1) I ;-\mu ; \lambda)$ are given as

$$
\begin{aligned}
& \mathcal{L}\{\mathcal{R}(w ;-n I, C+(n-1) I ; v ; \lambda)\}=\frac{1}{s^{\nu+1}} \mathcal{Y}_{n}(C ; \lambda,-s), \\
& \mathcal{L}\{\mathcal{R}(w ;-n I, C+(n-1) I ;-\mu ; \lambda)\}=\frac{1}{s^{\mu-1}} \mathcal{Y}_{n}(C ; \lambda,-s),
\end{aligned}
$$

where $\mathcal{Y}_{n}(C ; \lambda,-s)$ is the generalized Bessel matrix polynomial [9], and $n \in \mathbb{N}$.

Proof For $n \in \mathbb{N}$, replacing $A$ by $n I$ and $B$ by $C+(n-1) I$ in $\mathcal{R}(w ; A, B ; v ; \lambda)$ and then taking the Laplace transform of (17) yield

$$
\begin{aligned}
\mathcal{L} & \{\mathcal{R}(w ;-n I, C+(n-1) I ; v ; \lambda)\} \\
& =\mathcal{L}\left\{\frac{w^{v}}{\Gamma(v+1)}{ }_{2} R_{1}^{(1)}(-n I, C+(n-1) I ; v+1 ; \lambda w)\right\} \\
& =\int_{0}^{\infty} e^{-s w}\left\{\frac{w^{v}}{\Gamma(v+1)}{ }^{2} R_{1}^{(1)}(-n I, C+(n-1) I ; v+1 ; \lambda w)\right\} d w \\
& =\frac{1}{s^{v+1}} \sum_{k=0}^{n} \frac{(-n I)_{k}(C+(n-1) I)_{k}}{k !}\left(\frac{\lambda}{s}\right)^{k} \\
& =\frac{1}{s^{v+1}}{ }_{2} F_{0}\left(-n I, C+(n-1) I ;-;-\frac{\lambda}{(-s)}\right)=\frac{1}{s^{v+1}} \mathcal{Y}_{n}(C ; \lambda,-s) .
\end{aligned}
$$

This proves (25). Further,

$$
\begin{aligned}
\mathcal{L} & \{\mathcal{R}(w ;-n I, C+(n-1) I ;-\mu ; \lambda)\} \\
& =\mathcal{L}\left\{\frac{w^{-\mu}}{\Gamma(1-\mu)}{ }_{2} R_{1}^{(1)}(-n I, C+(n-1) I ; 1-\mu ; \lambda w)\right\} \\
& =\int_{0}^{\infty} e^{-s w}\left\{\frac{w^{-\mu}}{\Gamma(1-\mu)}{ }_{2} R_{1}^{(1)}(-n I, C+(n-1) I ; 1-\mu ; \lambda w)\right\} d w \\
& =\frac{1}{s^{1-\mu}} \sum_{k=0}^{n} \frac{(-n I)_{k}(C+(n-1) I)_{k}}{k !}\left(\frac{\lambda}{s}\right)^{k} \\
& =\frac{1}{s^{1-\mu}}{ }_{2} F_{0}\left(-n I, C+(n-1) I ;-;-\frac{\lambda}{(-s)}\right)=\frac{1}{s^{1-\mu}} \mathcal{Y}_{n}(C ; \lambda,-s) .
\end{aligned}
$$

This is the proof of (26).

From Theorems 3.1 and 3.2 we can obtain the following particular cases:

1. Taking $A=a \in \mathbb{C}^{1 \times 1}$ and $B=b \in \mathbb{C}^{1 \times 1}$ in Theorem 3.1, we get the classical results for the generalized hypergeometric function [22].

2. Choosing $C=c \in \mathbb{C}^{1 \times 1}$ in Theorem 3.2, we get the classical results for the generalized hypergeometric function [22]. 
4 Recurrence relation of ${ }_{2} \mathrm{R}_{1}^{(\tau)}(A, B ; C ; z)$

In this section, we give a matrix recurrence relation of Wright hypergeometric matrix function in the following theorem.

Theorem 4.1 Let $A$ and $B$ be positive stable matrices in $\mathbb{C}^{N \times N}$ with $B+n I$ invertible for all integers $n \geq 0$ and $|z|<1$. Then

$$
\begin{aligned}
(s+ & 1)_{2} \mathbf{R}_{1}^{(\tau)}(A, B ;(s+1) I ; z)-{ }_{2} \mathbf{R}_{1}^{(\tau)}(A, B ;(s+2) I ; z) \\
= & \left\{\frac{\tau^{2}}{(s+2)}\right\} z^{2} \frac{d^{2}}{d z^{2}}\left({ }_{2} \mathbf{R}_{1}^{(\tau)}(A, B ;(s+3) I ; z)\right)+z \frac{\tau}{(s+2)}\{\tau+2(s+1)\} \\
& \times \frac{d}{d z}\left({ }_{2} \mathbf{R}_{1}^{(\tau)}(A, B ;(s+3) I ; z)\right)+s_{2} \mathbf{R}_{1}^{(\tau)}(A, B ;(s+3) I ; z), \quad \operatorname{Re}(s)>0 .
\end{aligned}
$$

Proof Applying the fundamental relation of the gamma matrix function in (2) to (9), we can write

$$
\begin{aligned}
{ }_{2} \mathbf{R}_{1}^{(\tau)}(A, B ;(s+1) I ; z)= & \Gamma((s+1) I) \Gamma^{-1}(B) \\
& \times \sum_{n=0}^{\infty}(A){ }_{n} \Gamma(B+\tau n) \Gamma^{-1}((s+\tau n) I) \frac{z^{n}}{(s+\tau n) n !}
\end{aligned}
$$

and

$$
\begin{aligned}
&{ }_{2} \mathbf{R}_{1}^{(\tau)}(A, B ;(s+2) I ; z) \\
&= \Gamma((s+2) I) \Gamma^{-1}(B) \\
& \quad \times \sum_{n=0}^{\infty}(A)_{n} \Gamma(B+\tau n) \Gamma^{-1}((s+\tau n) I) \frac{z^{n}}{(s+1+\tau n)(s+\tau n) n !} .
\end{aligned}
$$

We can write equation (29) as

$$
\begin{aligned}
{ }_{2} \mathbf{R}_{1}^{(\tau)} & (A, B ;(s+2) I ; z) \\
= & \Gamma((s+2) I) \Gamma^{-1}(B) \\
& \times \sum_{n=0}^{\infty}\left\{\frac{1}{(s+\tau n)}-\frac{1}{(s+1+\tau n)}\right\}(A)_{n} \Gamma(B+\tau n) \Gamma^{-1}((s+\tau n) I) \frac{z^{n}}{n !} \\
= & (s+1)_{2} \mathbf{R}_{1}^{(\tau)}(A, B ;(s+1) I ; z)-\Gamma((s+2) I) \Gamma^{-1}(B) \\
& \times \sum_{n=0}^{\infty}(A)_{n} \Gamma(B+\tau n) \Gamma^{-1}((s+\tau n) I) \frac{z^{n}}{(s+1+\tau n) n !} .
\end{aligned}
$$

For our convenience, we denote the last summation in (30) by $\mathcal{S}$ :

$$
\begin{aligned}
\mathcal{S}= & \Gamma((s+2) I) \Gamma^{-1}(B) \\
& \times \sum_{n=0}^{\infty}(A)_{n} \Gamma(B+\tau n) \Gamma^{-1}((s+\tau n) I) \frac{z^{n}}{(s+1+\tau n) n !}
\end{aligned}
$$




$$
=(s+1){ }_{2} \mathbf{R}_{1}^{(\tau)}(A, B ;(s+1) I ; z)-{ }_{2} \mathbf{R}_{1}^{(\tau)}(A, B ;(s+2) I ; z) .
$$

Applying the simple identity $\frac{1}{\eta}=\frac{1}{\eta(\eta+1)}+\frac{1}{\eta+1}(\eta=s+1+\tau n)$ to (31), we obtain

$$
\begin{aligned}
\mathcal{S}= & \Gamma((s+2) I) \Gamma^{-1}(B) \\
& \times \sum_{n=0}^{\infty}((s+\tau n) I)(A)_{n} \Gamma(B+\tau n) \Gamma^{-1}((s+2+\tau n) I) \frac{z^{n}}{n !} \\
+ & \Gamma((s+2) I) \Gamma^{-1}(B) \\
& \times \sum_{n=0}^{\infty}((s+2+\tau n) I)(A)_{n} \Gamma(B+\tau n) \Gamma^{-1}((s+3+\tau n) I) \frac{z^{n}}{n !}
\end{aligned}
$$

and

$$
\begin{aligned}
\mathcal{S}= & \frac{\tau}{(s+2)}\left\{\Gamma((s+3) I) \Gamma^{-1}(B) \sum_{n=1}^{\infty}(A)_{n} \Gamma(B+\tau n) \Gamma^{-1}((s+3+\tau n) I) \frac{z^{n}}{(n-1) !}\right\} \\
& +\frac{s}{(s+2)}\left\{\Gamma((s+3) I) \Gamma^{-1}(B) \sum_{n=0}^{\infty}(A)_{n} \Gamma(B+\tau n) \Gamma^{-1}((s+3+\tau n) I) \frac{z^{n}}{n !}\right\} \\
& +\frac{\tau^{2}}{(s+2)}\left\{\Gamma((s+3) I) \Gamma^{-1}(B) \sum_{n=1}^{\infty}(A)_{n} \Gamma(B+\tau n) \Gamma^{-1}((s+3+\tau n) I) \frac{n z^{n}}{(n-1) !}\right\} \\
& +\frac{\tau(2 s+1)}{(s+2)}\left\{\Gamma((s+3) I) \Gamma^{-1}(B) \sum_{n=1}^{\infty}(A)_{n} \Gamma(B+\tau n) \Gamma^{-1}((s+3+\tau n) I) \frac{z^{n}}{(n-1) !}\right\} \\
& +\frac{s(s+1)}{(s+2)}\left\{\Gamma((s+3) I) \Gamma^{-1}(B) \sum_{n=1}^{\infty}(A)_{n} \Gamma(B+\tau n) \Gamma^{-1}((s+3+\tau n) I) \frac{z^{n}}{n !}\right\} .
\end{aligned}
$$

We now express each summation in the right-hand side of (33) as follows:

$$
\begin{aligned}
& \frac{d^{2}}{d z^{2}}\left(z_{2}^{2} \mathbf{R}_{1}^{(\tau)}(A, B ;(s+3) I ; z)\right) \\
& =2{ }_{2} \mathbf{R}_{1}^{(\tau)}(A, B ;(s+3) I ; z)+4 z \frac{d}{d z}{ }_{2} \mathbf{R}_{1}^{(\tau)}(A, B ;(s+3) I ; z) \\
& \quad+z^{2} \frac{d^{2}}{d z^{2}}{ }_{2} \mathbf{R}_{1}^{(\tau)}(A, B ;(s+3) I ; z)
\end{aligned}
$$

and

$$
\begin{aligned}
& \frac{d^{2}}{d z^{2}}\left(z_{2}^{2} \mathbf{R}_{1}^{(\tau)}(A, B ;(s+3) I ; z)\right) \\
& =\Gamma((s+3) I) \Gamma^{-1}(B) \sum_{n=0}^{\infty}(A)_{n} \Gamma(B+\tau n) \Gamma^{-1}((s+3+\tau n) I) \frac{(n+2)(n+1) z^{n}}{n !} \\
& =\Gamma((s+3) I) \Gamma^{-1}(B) \sum_{n=0}^{\infty}(A)_{n} \Gamma(B+\tau n) \Gamma^{-1}((s+3+\tau n) I) \frac{n z^{n}}{(n-1) !} \\
& \quad+3 \Gamma((s+3) I) \Gamma^{-1}(B) \sum_{n=0}^{\infty}(A)_{n} \Gamma(B+\tau n) \Gamma^{-1}((s+3+\tau n) I) \frac{z^{n}}{(n-1) !}
\end{aligned}
$$




$$
+2{ }_{2} \mathbf{R}_{1}^{(\tau)}(A, B ;(s+3) I ; z)
$$

From (34) and (35) we get

$$
\begin{aligned}
\Gamma((s+3) I) \Gamma^{-1}(B) \sum_{n=1}^{\infty}(A)_{n} \Gamma(B+\tau n) \Gamma^{-1}((s+3+\tau n) I) \frac{n z^{n}}{(n-1) !} \\
=z^{2} \frac{d^{2}}{d z^{2}}{ }_{2} \mathbf{R}_{1}^{(\tau)}(A, B ;(s+3) I ; z) \\
\quad+4 z \frac{d}{d z}{ }_{2} \mathbf{R}_{1}^{(\tau)}(A, B ;(s+3) I ; z) \\
\quad-3 \Gamma((s+3) I) \Gamma^{-1}(B) \sum_{n=1}^{\infty}(A)_{n} \Gamma(B+\tau n) \Gamma^{-1}((s+3+\tau n) I) \frac{z^{n}}{(n-1) !} .
\end{aligned}
$$

Suppose that

$$
\begin{aligned}
& \frac{d}{d z}\left(z_{2} \mathbf{R}_{1}^{(\tau)}(A, B ;(s+3) I ; z)\right) \\
& \quad={ }_{2} \mathbf{R}_{1}^{(\tau)}(A, B ;(s+3) I ; z)+z \frac{d}{d z}{ }_{2} \mathbf{R}_{1}^{(\tau)}(A, B ;(s+3) I ; z)
\end{aligned}
$$

and

$$
\begin{aligned}
& \frac{d}{d z}\left(z_{2} \mathbf{R}_{1}^{(\tau)}(A, B ;(s+3) I ; z)\right) \\
& =\Gamma((s+3) I) \Gamma^{-1}(B) \sum_{n=0}^{\infty}(A)_{n} \Gamma(B+\tau n) \Gamma^{-1}((s+3+\tau n) I) \frac{(n+1) z^{n}}{n !} \\
& =\Gamma((s+3) I) \Gamma^{-1}(B) \sum_{n=1}^{\infty}(A)_{n} \Gamma(B+\tau n) \Gamma^{-1}((s+3+\tau n) I) \frac{z^{n}}{(n-1) !} \\
& \quad+{ }_{2} \mathbf{R}_{1}^{(\tau)}(A, B ;(s+3) I ; z) .
\end{aligned}
$$

From (37) and (38) we get

$$
\begin{aligned}
& \Gamma((s+3) I) \Gamma^{-1}(B) \sum_{n=1}^{\infty}(A)_{n} \Gamma(B+\tau n) \Gamma^{-1}((s+3+\tau n) I) \frac{z^{n}}{(n-1) !} \\
& \quad=z \frac{d}{d z}{ }_{2} \mathbf{R}_{1}^{(\tau)}(A, B ;(s+3) I ; z) .
\end{aligned}
$$

Combining (36) and (39) yields

$$
\begin{aligned}
\Gamma((s+3) I) \Gamma^{-1}(B) \sum_{n=1}^{\infty}(A)_{n} \Gamma(B+\tau n) \Gamma^{-1}((s+3+\tau n) I) \frac{n z^{n}}{(n-1) !} \\
=z^{2} \frac{d^{2}}{d z^{2}}{ }_{2} \mathbf{R}_{1}^{(\tau)}(A, B ;(s+3) I ; z) \\
\quad+z \frac{d}{d z}{ }_{2} \mathbf{R}_{1}^{(\tau)}(A, B ;(s+3) I ; z) .
\end{aligned}
$$


Now applying (39) and (40) to (33), we get

$$
\begin{aligned}
\mathcal{S}= & \left\{\frac{\tau^{2}}{(s+2)}\right\} z^{2} \frac{d^{2}}{d z^{2}}{ }_{2} \mathbf{R}_{1}^{(\tau)}(A, B ;(s+3) I ; z) \\
& +\left\{\frac{\tau^{2}+\tau+\tau(2 s+1)}{(s+2)}\right\} z \frac{d}{d z}{ }_{2} \mathbf{R}_{1}^{(\tau)}(A, B ;(s+3) I ; z) \\
& +\frac{s+s(s+1)}{s+2}{ }_{2} \mathbf{R}_{1}^{(\tau)}(A, B ;(s+3) I ; z) .
\end{aligned}
$$

From (30), (31), and (41) we arrive at

$$
\begin{aligned}
(s+ & 1)_{2} \mathbf{R}_{1}^{(\tau)}(A, B ;(s+1) I ; z)-{ }_{2} \mathbf{R}_{1}^{(\tau)}(A, B ;(s+2) I ; z) \\
= & \left\{\frac{\tau^{2}}{(s+2)}\right\} z^{2} \frac{d^{2}}{d z^{2}}\left({ }_{2} \mathbf{R}_{1}^{(\tau)}(A, B ;(s+3) I ; z)\right)+z \frac{\tau}{(s+2)}\{\tau+2(s+1)\} \\
& \times \frac{d}{d z}\left({ }_{2} \mathbf{R}_{1}^{(\tau)}(A, B ;(s+3) I ; z)\right)+s_{2} \mathbf{R}_{1}^{(\tau)}(A, B ;(s+3) I ; z), \quad s>0 .
\end{aligned}
$$

This completes the proof of the theorem.

Remark For $\tau=1$, result (27) reduces to the result for Gauss hypergeometric matrix function [9].

Remark Taking $A=a \in \mathbb{C}^{1 \times 1}, B=b \in \mathbb{C}^{1 \times 1}, C=c \in \mathbb{C}^{1 \times 1}$ in Theorem 4.1, we easily obtain the known result derived by Rao et al. [22].

Remark Taking $A=a \in \mathbb{C}^{1 \times 1}, B=b \in \mathbb{C}^{1 \times 1}, C=c \in \mathbb{C}^{1 \times 1}$, and $\tau=1$ in Theorem 4.1, we obtain the recurrence relation for the classical Gauss hypergeometric function [2].

\section{Integral formulas of ${ }_{2} R_{1}^{(\tau)}(A, B ; C ; Z)$}

In this section, we give integral formulas of the Wright hypergeometric matrix function by the following theorem.

Theorem 5.1 Let $A, B$, and $C$ be positive stable matrices in $\mathbb{C}^{N \times N}$ satisfying condition (5). Then for $\tau>0$ and $m>0$, we have the following integrals:

$$
\begin{aligned}
& \int_{0}^{\infty} \exp \left(\frac{-u^{m}}{z^{m}}\right) u^{C-(\tau+1) I}\left[\sum_{n=0}^{\infty}(A)_{n} \Gamma(B+\tau n I) \Gamma(C)\right. \\
& \left.\quad \times \Gamma^{-1}(B) \Gamma^{-1}(C+\tau n I) \Gamma^{-1}\left(\frac{C-(\tau+n) I}{m}\right) u^{n}\right] d u \\
& =\frac{z^{C-\tau I}}{m}{ }_{2} \mathbf{R}_{1}^{(\tau)}(A, B ; C ; z), \quad|z|<1, \\
& \int_{0}^{1} z^{m}{ }_{2} \mathbf{R}_{1}^{(\tau)}\left(A, B ; m I ; z^{\tau}\right) d z \\
& =\frac{{ }_{2} \mathbf{R}_{1}^{(\tau)}(A, B ;(m+1) I ; 1)}{m}
\end{aligned}
$$




$$
-\frac{{ }_{2} \mathbf{R}_{1}^{(\tau)}(A, B ;(m+2) I ; 1)}{m(m+1)}, \quad\left|z^{\tau}\right|<1
$$

Proof For convenience, denote the left-hand side of (43) by $\mathcal{T}$. Substituting $\frac{-u^{m}}{z^{m}}=\zeta$, we obtain

$$
\begin{aligned}
\mathcal{T}= & \int_{0}^{\infty} \exp (-\zeta) z^{C-(\tau+1) I} \zeta^{\frac{C-(\tau+1) I}{m}}\left\{\sum_{n=0}^{\infty} \frac{(A)_{n}}{n !} \Gamma(B+\tau n I) \Gamma(C)\right. \\
& \left.\times \Gamma^{-1}(B) \Gamma^{-1}(C+\tau n I) \Gamma^{-1}\left(\frac{C-(\tau-n) I}{m}\right) z^{n} \zeta^{\frac{n}{m}}\right\} \frac{z}{m} \zeta^{\frac{1-m}{m}} d \zeta \\
= & \frac{z^{C-\tau I}}{m}\left\{\sum_{n=0}^{\infty} \frac{(A)_{n}}{n !} \Gamma(B+\tau n I) \Gamma(C) \Gamma^{-1}\left(\frac{C-(\tau-n) I}{m}\right)\right. \\
& \left.\times \Gamma^{-1}(B) \Gamma^{-1}(C+\tau n I) \Gamma\left(\frac{C-(\tau-n) I}{m}\right) z^{n}\right\} .
\end{aligned}
$$

Further simplification yields

$$
\mathcal{T}=\frac{z^{C-\tau I}}{m}{ }_{2} \mathbf{R}_{1}^{(\tau)}(A, B ; C ; z)
$$

which evidently leads us to the required result in (43).

Now putting $z=1$ in (31) yields

$$
\begin{aligned}
\Gamma(m I) \Gamma^{-1}(B) & \left\{\sum_{n=0}^{\infty} \frac{(A)_{n}}{n !(m+1+\tau n)} \Gamma(B+\tau n I) \Gamma^{-1}((m+\tau n) I)\right. \\
= & \frac{{ }_{2} \mathbf{R}_{1}^{(\tau)}(A, B ;(m+1) I ; 1)}{m} \\
& \left.-\frac{{ }_{2} \mathbf{R}_{1}^{(\tau)}(A, B ;(m+2) I ; 1)}{m(m+1)}\right\} .
\end{aligned}
$$

Suppose that

$$
\begin{aligned}
& \int_{0}^{t} z^{m}{ }_{2} \mathbf{R}_{1}^{(\tau)}\left(A, B ; m I ; z^{\tau}\right) d z \\
& \quad=\int_{0}^{t} z^{\tau} \Gamma(m I) \Gamma^{-1}(B)\left\{\sum_{n=0}^{\infty} \frac{(A)_{n} z^{\tau n}}{n !} \Gamma(B+\tau n I) \Gamma^{-1}((m+\tau n) I)\right\} d z \\
& \quad=\Gamma(m I) \Gamma^{-1}(B)\left\{\sum_{n=0}^{\infty} \frac{(A)_{n} t^{m+1+\tau n}}{n !(m+1+\tau n)} \Gamma(B+\tau n I) \Gamma^{-1}((m+\tau n) I)\right\} .
\end{aligned}
$$

Now, comparing (47) and (48) after setting $t=1$ in (48), we have

$$
\begin{array}{r}
\int_{0}^{1} z^{m}{ }_{2} \mathbf{R}_{1}^{(\tau)}\left(A, B ; m I ; z^{\tau}\right) d z \\
=\frac{{ }_{2} \mathbf{R}_{1}^{(\tau)}(A, B ;(m+1) I ; 1)}{m}
\end{array}
$$




$$
-\frac{{ }_{2} \mathbf{R}_{1}^{(\tau)}(A, B ;(m+2) I ; 1)}{m(m+1)} .
$$

This is proves (44) in Theorem 5.1.

Remark For $\tau=1$, results (43) and (44) reduce to integral representations for the Gauss hypergeometric matrix function (see $[7,9])$.

\section{Conclusions}

The generalized (Wright) hypergeometric function was first studied by Virchenko et al. [23] as follows:

$$
{ }_{2} \mathbf{R}_{1}^{(\tau)}(a, b ; c ; z)=\frac{\Gamma(c)}{\Gamma(b)} \sum_{n=0}^{\infty} \frac{(a)_{n} \Gamma(b+\tau n)}{\Gamma(c+\tau n)} \frac{z^{n}}{n !}, \quad \tau>0,|z|<1 .
$$

Later on, many authors (see. e.g., [24-26]) introduced several extensions of the generalized (Wright) hypergeometric function. Very recently, the Wright hypergeometric matrix functions and incomplete Wright Gauss hypergeometric matrix functions were introduced by Bakhet et al. [1]. Here, with the help of the well-known fractional operators, we have obtained the Wright-type hypergeometric matrix functions and its properties. Also, we presented some properties of the Wright hypergeometric matrix function such as matrix recurrence relations and integral representations. Further research on this topic is now under investigation and will be reported in forthcoming papers.

\section{Acknowledgements}

The authors extend their appreciation to the Deanship of Scientific Research at King Khalid University for funding this work through General Research Project under Grant No. (G.R.P-172/41).

\section{Funding}

Deanship of Scientific Research at King Khalid University.

\section{Availability of data and materials}

The data that support the findings of this study are available from the authors upon request.

\section{Competing interests}

The authors declare that they have no competing interests.

\section{Authors' contributions}

All authors jointly worked on the results, and they read and approved the final manuscript.

\section{Publisher's Note}

Springer Nature remains neutral with regard to jurisdictional claims in published maps and institutional affiliations.

Received: 21 February 2020 Accepted: 18 May 2020 Published online: 27 May 2020

\footnotetext{
References

1. Bakhet, A., Jiao, Y., He, F.: On the Wright hypergeometric matrix functions and their fractional calculus. Integral Transforms Spec. Funct. 30, 138-156 (2019)

2. Seaborn, J.: Hypergeometric Functions and Their Applications. Springer, New York (1991)

3. Agarwal, P., Agarwal, R.P., Ruzhansky, M.: Special Functions and Analysis of Differential Equations, 1st edn. CRC Press, Boca Raton (2020)

4. Akhmedova, V., Akhmedov, E.: Selected Special Functions for Fundamental Physics. SpringerBriefs in Physics (2019)

5. Ruzhansky, M., JeCho, Y., Agarwal, P., Area, l.: Advances in Real and Complex Analysis with Applications (Trends in Mathematics). Birkhäuser, Basel (2017)

6. Agarwal, P., Al-Mdallal, Q., Cho, Y., Shilpi, J.: Fractional differential equations for the generalized Mittag-Leffler function. Adv. Differ. Equ. 2018, 58 (2018)

7. Jódar, L., Cortés, J.: On the hypergeometric matrix function. J. Comput. Appl. Math. 99, 205-217 (1998)

8. Tirao, J.: The matrix-valued hypergeometric equation. Proc. Natl. Acad. Sci. USA 100, 8138-8141 (2003)
} 
9. Abdalla, M.: Special matrix functions: characteristics, achievements and future directions. Linear Multilinear Algebra 68, 1-28 (2020)

10. Hidan, M., Abdalla, M.: A note on the Appell hypergeometric matrix function. Math. Probl. Eng. 2020, Article ID 6058987 (2020)

11. He, F., Bakhet, A., Hidan, M., Abdalla, M.: On the extended hypergeometric matrix functions and their applications for the derivatives of the extended Jacobi matrix polynomial. Math. Probl. Eng. 2020, Article ID 4268361 (2020)

12. Abdalla, M.: Further results on the generalized hypergeometric matrix functions. Int. J. Comput. Math. Sci. 10, 1-10 (2019)

13. Abdalla, M., Bakhet, A.: Extended Gauss hypergeometric matrix functions. Iran. J. Sci. Technol. Trans. A, Sci. 42, $1465-1470(2018)$

14. Abdalla, M.: On the incomplete hypergeometric matrix functions. Ramanujan J. 43, 663-678 (2017)

15. Bakhet, A., He, F.: On 2-variables Konhauser matrix polynomials and their fractional integrals. Mathematics 8, 232 (2020)

16. Zayed, M., Abul-Ez, M., Abdalla, M., Saeed, N.: On the fractional order Rodrigues formula for the shifted Legendre-type matrix polynomials. Mathematics 8, 136 (2020)

17. He, F., Bakhet, A., Hidan, M., Abdalla, M.: Two variables Shivley's matrix polynomials. Symmetry 11, 151 (2019)

18. Cortęs, J., Jodar, L., Sols, F., Carrillo, R.: Infinite matrix products and the representation of the gamma matrix function. Abstr. Appl. Anal. 2015, Article ID 564287 (2015)

19. Agarwal, P., Baleanu, D., Chen, Y., Momani, S., Machado, J.: Fractional Calculus: ICFDA 2018, Amman. Springer Proceedings in Mathematics Statistics, vol. 303 (2020)

20. Abdalla, M., Hidan, M.: Fractional orders of the generalized Bessel matrix polynomials. Eur. J. Pure Appl. Math. 10, 995-1004 (2017)

21. Debnath, L., Bhatta, D.: Integral Transform and Their Application. CRC Press, Boca Raton (2015)

22. Rao, S., Prajapati, J., Patel, A., Shukla, A.: Some properties of generalized hypergeometric function. Commun. Korean Math. Soc. 28, 303-317 (2013)

23. Virchenko, N., Kalla, S., Al-Zamel, A.: Some results on a generalized hypergeometric function. Integral Transforms Spec. Funct. 12, 89-100 (2001)

24. Ghaffar, A., Saif, A., Iqbal, M., Rizwan, M.: Two classes of integrals involving extended Wright type generalized hypergeometric function. Commun. Math. Appl. 10, 599-606 (2019)

25. Safdar, M., Rahman, G., Ullah, Z., Ghaffar, A., Nisar, K.: A new extension of the Pochhammer symbol and its application to hypergeometric functions. Int. J. Appl. Comput. Math. 5, 151 (2019)

26. Srivastava, H., Tassaddiq, A., Rahman, G., Nisar, K., Khan, l.: A new extension of the t-Gauss hypergeometric function and its associated properties. Mathematics 7, 996 (2019)

\section{Submit your manuscript to a SpringerOpen ${ }^{\circ}$ journal and benefit from:}

- Convenient online submission

- Rigorous peer review

- Open access: articles freely available online

- High visibility within the field

- Retaining the copyright to your article

Submit your next manuscript at $\boldsymbol{~ s p r i n g e r o p e n . c o m ~}$ 